\begin{tabular}{|l|l|l|}
\hline J. & Julien & Bogousslavsky \\
\hline M.G. & Michel G. & Hennerici \\
\hline
\end{tabular}

\title{
Yet Another Journal?
}

Together with the Editorial Board and the Advisory Board, we are happy to introduce to you the first issue of Cerebrovascular Diseases. Why another Journal, when it is already more and more difficult to keep up with the fastly growing literature in the already existing Journals? Just because it has become obvious to many investiga-tors involved in cerebrovascular disease and stroke that the existing Journals do not provide a sufficient forum in this field, which is one of the most rapidly expanding areas of research in the clinical medical sciences. While the trend in many Journals is to emphasize experimental studies, the aim of Cerebrovascular Diseases is to provide the readership with original and relevant works on all clinical aspects related to cerebrovascular diseases and stroke. Because the field of cerebrovascular diseases en-compasses varied specialties, such as neurology, internal medicine, surgery, radiology, epidemiology, cardiology, hematology, psychology, or rehabilitation, we are confi-dent that Cerebrovascular Diseases will be a Journal of

synthesis and alliances of opinions, be they consensus or controversy, instead of a catalogue of hyperspecialized articles.

All papers, from short case reports to original articles or reviews, have been and will be subjected to peer review before acceptance for publication or rejection, in order to offer high Standard papers to the readers from the very first issues of Cerebrovascular Diseases.

T. Together with S. Karger AG

we have worked to provide a truly international Journal supported by international boards. It is becoming clear that papers are already submitted from all continents. We hope

in this time of loosening boundaries

that this new Journal will contribute to tighten up the scientific Community involved in the care of stroke patients and in the study of cerebrovascular diseases.

Julien Bogousslavsky Michael G. Hennerici 